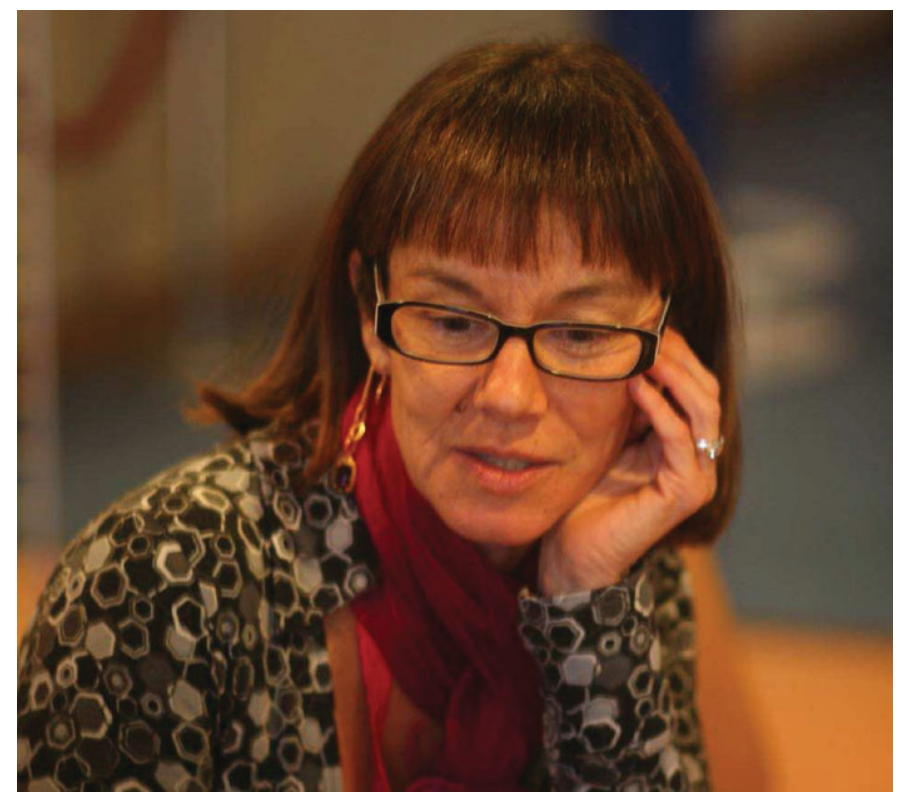

Sofia Randich

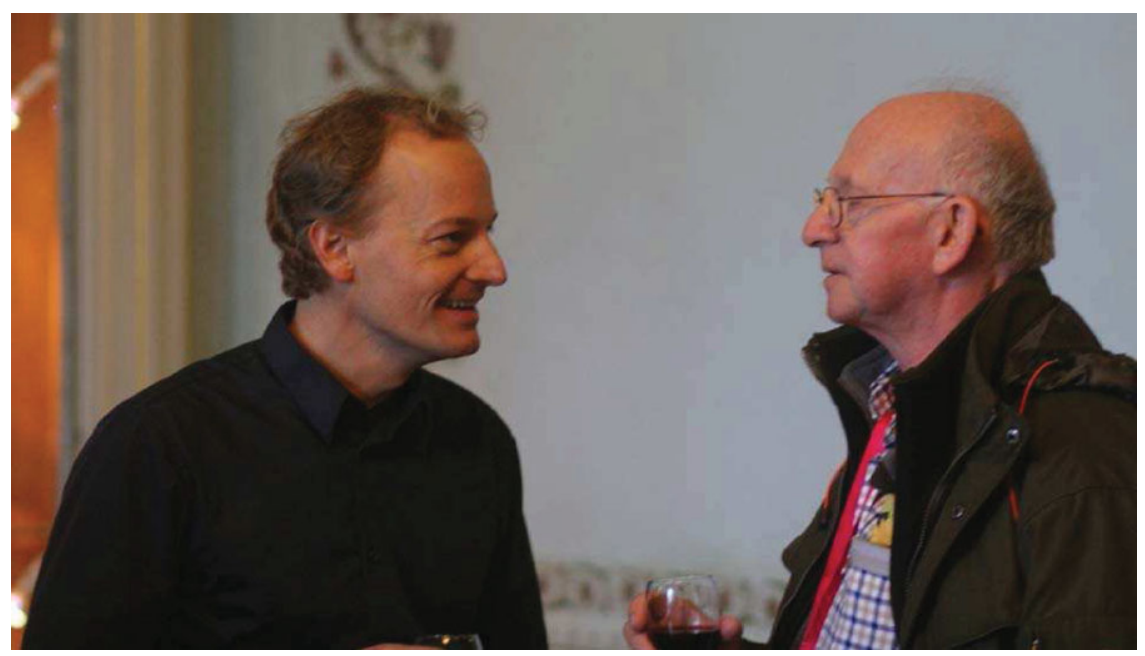

Andreas Korn \& Jeffrey Linsky 


\title{
Mass loss and luminosities of S and C AGB stars with and without $\mathrm{Li}$
}

\author{
Roald Guandalini $^{1}$, Sara Palmerini ${ }^{1,2}$, Maurizio Busso ${ }^{1,2}$, Enrico \\ Maiorca $^{1,2}$, and Stefan Uttenthaler ${ }^{3}$ \\ ${ }^{1}$ Dipartimento di Fisica, Universitá degli Studi di Perugia, \\ via Pascoli, 06123, Perugia, Italy \\ email: guandalini@fisica.unipg.it \\ ${ }^{2}$ I.N.F.N. sezione di Perugia \\ ${ }^{3}$ Instituut voor Sterrenkunde, K. U. Leuven, \\ Celestijnenlaan 200D, 3000 Leuven, Belgium
}

\begin{abstract}
We present the preliminary results of an analysis performed on two samples of thermally pulsing Asymptotic Giant Branch stars from our Galaxy, the first made of carbonrich sources and the second of S-type stars. We have estimated their absolute luminosities and updated rates of the stellar winds through methods based on their infrared spectrophotometry and on updated estimates of their variability and distance.

We then focus on those sources in our database showing $\mathrm{Li}$ in their spectra looking for correlations between the $\mathrm{Li}$ abundance and the other physical parameters, in the aim of establishing observational criteria for understanding the conditions for the occurrence of the deep mixing phenomena to which the production of $\mathrm{Li}$ is currently attributed.
\end{abstract}

Keywords. Stars: evolution, AGB, post-AGB - infrared: stars

\section{Introduction on $\mathrm{Li}$ and evolved giant stars}

Stars evolving along the red giant branch are on average depleted in lithium. We remind here that, for low-metallicity objects, an upper limit on the Li content was set by Gratton et al. (2000), stating that low-mass stars on the upper RGB have log $\epsilon(\mathrm{Li})$ $\leqslant 0$. Mixing processes on the Main Sequence and up to the first dredge-up imply that Li be gradually carried down from the photosphere to regions of high temperature and destroyed; at the same time new $\mathrm{Li}$ is not produced, because any mixing mechanism occurring is so slow that the parent nucleus ${ }^{7}$ Be has time to capture protons on the path. This explains why red giants are mainly Li-poor. It was further established (Sweigart \& Mengel 1979) and confirmed by observations (Gilroy 1989, Gilroy \& Brown 1991, Kraft 1994) that there must be slow mixing episodes in low-mass stars also after the first dredge-up. They gradually carry to the surface a considerable amount of ${ }^{13} \mathrm{C}$, so that the ${ }^{12} \mathrm{C} /{ }^{13} \mathrm{C}$ ratio decreases. These mixing phenomena can happen because after dredgeup, the H-burning shell advances into a homogeneous region, so that the natural barrier opposed to mass transport by a chemical stratification is not present. This happens in the so-called "L-bump phase". Slow mixing processes occurring at or after the L-bump would further reduce the Li abundance. However, a few red giants (about $2 \%$ ) show enhanced Li abundances at levels sometimes higher than in the present Interstellar Medium $(\log \epsilon(\mathrm{Li})$ $\simeq 3.3$ ). Amongst the various explanations attempted, the most common assumption is that some form of fast extra-mixing might transport ${ }^{7} \mathrm{Be}$ from above the H-burning shell to the envelope at a speed sufficient to overcome the rate of p-captures. This is the so-called Cameron-Fowler mechanism (Cameron \& Fowler 1971). The attempts at 
involving stellar rotation as source for these extra-mixing processes were frustrated by the understanding that the stellar structure reacts to rotational distortions too quickly to induce significant mixing on long time scales (Palacios et al. 2006).

It has been suggested (Busso et al. 2007a, Wasserburg \& Busso 2008, Nordhaus et al. 2008) that the processes that cause the mass transport might be linked to the buoyancy of magnetized H-burning ashes, based on the fact that magnetic bubbles are lighter than the stellar environment, due to the unbalance generated by magnetic pressure $\left(B^{2} / 8 \pi\right)$. This requires a magnetic dynamo to occur in red giants, as indeed observed (Andrews et al. 1988). Magnetic buoyancy is an interesting possibility especially because it can occur at different speeds, depending on the amount of heat exchanged with the environment (Denissenkov et al. 2009, Palmerini \& Busso 2008). Therefore, Li could experience production or destruction according to different mixing velocities.

In order to perform an accurate analysis on $\mathrm{Li}$ abundances and to attribute them to the correct evolutionary phases, we need reliable estimates of absolute luminosities and mass loss rates. Therefore, we here apply bolometric corrections for evolved stars, as derived in our recent work, to infer reliable estimates of these parameters (and of the distance) for stars having measurements of $\mathrm{Li}$ abundances in their photosphere. This helps in setting constraints on where extra-mixing is active. In Section 2 we discuss the analysis we performed and link it with our models. Instead, in Section 3 preliminary conclusions are illustrated.

\section{Luminosities and mass loss rates of red giants showing $\mathbf{L i}$}

\subsection{The AGB sub-sample}

In order to improve our understanding of the evolutionary properties of Asymptotic Giant Branch stars (hereafter AGB) we need to study in an accurate way two crucial parameters: luminosities and mass loss rates. For both, determinations are hampered by the large distance uncertainties and by the fact that most of the radiation emitted by these sources can be observed only at infrared wavelengths and therefore depends on space-borne facilities, or on the exploitation of special ground-based locations.

In the last years we performed an extensive analysis of the photometric properties of AGB stars, using available infrared data from the ISO, MSX and IRAS-LRS experiments and looking for relations between their luminosities and their main chemical and physical parameters. In this way we are trying to find crucial tools that could help us in the improvement of the evolutionary models for AGB sources.

In the particular case of Li-bearing stars the formation of our sub-sample starts from the previous work by Uttenthaler et al. (2007) on AGB stars in the Galactic bulge with Li. We extend it to the Galactic AGB stars of the disk, already examined in their photometrical properties in our previous works (Guandalini et al. 2006, Guandalini \& Busso 2008), with the aim of creating a homogeneous sub-sample with good estimates for mass and Li-abundances, good measurements of the distance and reliable near-tomid infrared photometry. The resulting list of sources and all the details on the analysis performed are presented in Guandalini et al. (2009; Table 1). All sources come from large samples of C-rich or S-type AGB stars; the photometric data used and the techniques adopted are from Guandalini \& Busso (2008), Busso et al. (2007b), and Guandalini et al. (2006).

\subsection{Luminosities and mass loss rates}

Figure 1 presents the selected sample of AGB stars showing Li in their spectra as compared with the luminosities obtained through the bolometric corrections from Guandalini 
et al. (2006) [C-rich] and Guandalini \& Busso (2008) [S-type]. The sources richest in Li are all of CJ type: we believe, also thanks to our analysis performed on C-rich galactic sources in Guandalini et al. (2006), that they are all source with peculiar evolutionary properties. Their high Li abundance can be produced either by a relatively fast extramixing in low-mass stars or by hot bottom burning in more massive AGB stars. Going down along the axis of the Li-abundance we find the four stars from the Galactic bulge discussed by Uttenthaler et al. (2007): they are all O-rich. We have marked by dashed lines the region occupied by "standard" $\mathrm{C}(\mathrm{N})$ sources (circles). They are divided into two groups according to the higher (full circles) or lower (empty circles) reliability of their distance estimate. For this group we can see that there seems to be a pattern for which more luminous sources are more depleted in $\mathrm{Li}$ and fainter ones are instead less depleted. This could be originated by two concurring phenomena: 1) more luminous Carbon stars are on average more evolved, so had more time to destroy Li; 2) more luminous Carbon stars could be the most massive ones (in a relatively small range of masses), therefore they could destroy Li more efficiently due to higher H-burning temperatures. If we look at the vertical dashed lines, they indicate the range of the luminosity function for Galactic C-rich sources from Guandalini et al. (2006). Our stars belong to the typical luminosity range of normal $\mathrm{C}$-stars and cover all the interval, therefore we can conclude that our sample is a good representative for the standard galactic Carbon stars from the disk. We believe that also their Li abundances should represent typical trends. Finally, we see that the few S-type (or M) sources we have in our sample are more depleted in Li than the average standard Carbon star.

If we look at Figure 1 we see a pattern for which the more luminous C-rich AGBs are the more depleted in $\mathrm{Li}$. In order to study this property we have to consider in the picture also Red Giant Branch stars (hereafter RGB). Therefore, we add in the analysis a

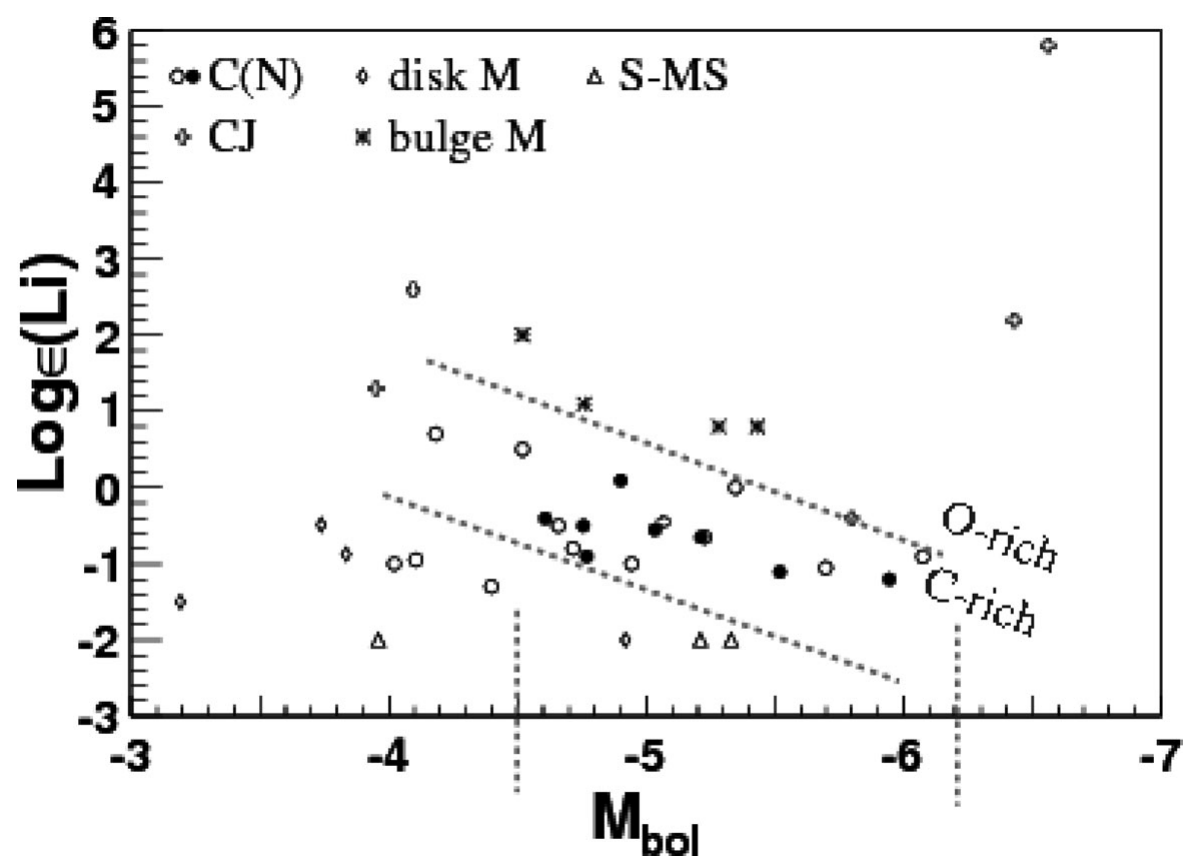

Figure 1. The sample of AGB stars showing Li in their spectra. Dashed lines roughly limit the region occupied by $\mathrm{C}(\mathrm{N})$ giants and their range of luminosities as inferred from the empirical Luminosity Function of Guandalini et al. (2006) (vertical lines). 
second sub-sample of RGB stars quite close to us, whose distances and Li-abundances are well-studied. The data for this group of K- and G-type sources are reported in Table 2 of Guandalini et al. (2009). In Figure 2 we consider both sub-samples in the same plot: RGB stars are the less luminous on the left side. The stars at the left of the plot, with $\log \varepsilon(\mathrm{Li})$ $\simeq 2$, are $\mathrm{K}$ giants with luminosities typical of the L-bump on the RGB. We consider them as being stars newly enriched in Li in agreement with Charbonnel \& Balachandran (2000). After the extensive depletion of Li on the Main Sequence and on the early RGB phases, their envelopes must have seen some form of rapid mixing implementing a CameronFowler mechanism. The second moderately Li-rich group is considered by us as being in the upper part of the RGB itself. Instead, Charbonnel \& Balachandran (2000) interpreted this group as formed by early-AGB stars, producing Li (after some destruction in the late RGB phases). Inspection of stellar models, however, reveals that the early-AGB stages suitable to reproduce the luminosities and temperatures of these $\mathrm{K}$ giants fall in a temporal phase where the $\mathrm{H}$-shell is extinguished, so that no ${ }^{7} \mathrm{Be}$ survives to be carried to the surface. We must therefore tentatively conclude that a new production of $\mathrm{Li}$ on the early-AGB at temperatures and luminosities compatible with observations does not occur and therefore the observed Li should be a relic of the previous production on the RGB. G-type stars show values of Li-abundance ranging between 0 and 1 . They are not totally depleted but don't show the high values of K-type Li-rich sources. Finally, Ktype sources without Li could be intermediate-mass stars with no hot bottom burning or low-mass stars with strong phenomena of depletion.

We searched also for possible relations between the rates of the stellar winds and Li-abundances for AGB stars. In order to estimate the rates, we adopted the methods explained in detail in Guandalini et al. (2006) and Guandalini (2010). Unfortunately, we didn't find any apparent relation between these two parameters: the data have a too large scatter to draw any conclusion.

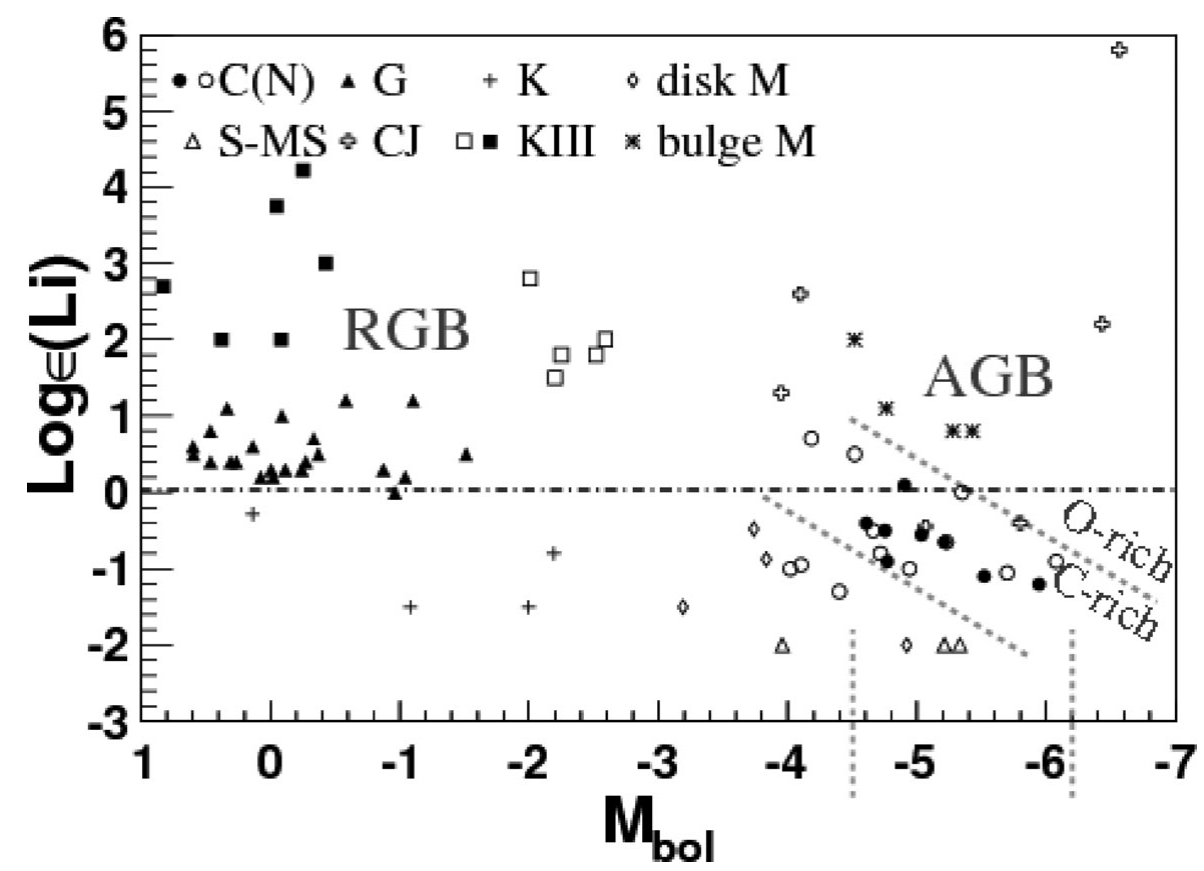

Figure 2. The same plot already presented in Figure 1, but including also the RGB sub-sample. See text and Guandalini et al. (2009) for details. 


\subsection{Link with the models}

Our interpretation of the above results can be summarized in the following points [see Palmerini et al. (2010), this conference, for more details on this part]:

1) We observe in Figures 1 and 2 that $\mathrm{Li}$ is produced at the Luminosity bump and then is depleted with different rates during the successive evolution of the sources along the RGB and AGB stages.

2) We have so far no clear evidence for another phase of production after the L-bump.

3) This could be explained by the model presented by Palmerini et al. (2009) that suggests two types of extra-mixing. i) Fast Mixing, due to the buoyancy of small magnetized parcels, generated by detached magnetic instabilities. This causes ${ }^{7}$ Be transport at rates faster than its decay lifetime (53 days) and therefore produces Li. This appears to happen at the bump of the Luminosity function following the first dredge-up. ii) Slow Mixing, due to the slower buoyancy of larger magnetized domains, which efficiently exchange heat with the environment, hence slowing down their motion. This happens during the remaining evolution and causes depletion of $\mathrm{Li}$.

\section{Conclusions}

We have examined a sub-sample of Galactic AGB stars of moderate luminosity (Bolometric Magnitude fainter than -6) having phenomena of extra-mixing for Li. The study of this sub-sample gives us the chance to refine our previous studies made on larger and more general samples thanks to their higher homogeneity and to the presence of another constraint: the Li-abundance.

The relation between the $\mathrm{Li}$ abundance and the luminosity, examined for different classes of stars along the RGB and the AGB branches, shows us that, for stars of moderate luminosity, $\mathrm{Li}$ is produced at the Bump of the L-function and then is depleted at different rates during the successive evolution of the sources.

No clear correlation of $\mathrm{Li}$ abundances with mass loss rates emerged so far.

The behaviour of $\mathrm{Li}$ in evolved stars seems to be explained by the model presented in Palmerini et al. (2010), this conference, thanks to two different phenomena of extramixing:

- Fast Mixing, at the bump of the L-function, causing production of Li (buoyancy, at speeds close to the Alfvén Velocity, of small bubbles formed by magnetic instabilities).

- Slow Mixing, during the remaining evolution, which causes depletion of Li (buoyancy of larger magnetized structures exchancing heat efficiently with the environment).

\section{Acknowledgments}

This research was supported by the Italian Ministry of Research under contract PRIN2006-022731 and by INFN (sezione di Perugia) through the funds of Research Group n. 3. The idea of mixing by magnetic buoyancy was developed in common with G. J. Wasserburg and K. M. Nollett. R. G. wishes to thank the organizers of IAU Symposium 268 for the chance of giving a talk and for the wonderful conference.

\section{References}

Andrews, A. D., Rodonó, M., Lensky, J. L. et al. 1988, A\&A, 204, 177

Busso, M., Wasserburg, G. J., Nollett, K. M., \& Calandra, A. 2007a, ApJ, 671, 802

Busso, M., Guandalini, R., Persi, P., Corcione, L., \& Ferrari-Toniolo, M. 2007b, AJ, 133, 2310

Cameron, A. G. W. \& Fowler, W. A. 1971, ApJ, 164, 111 
Charbonnel, C. \& Balachandran, S. C. 2000, A\& $A, 359,563$

Denissenkov, P. A., Pinsonneault, M., \& Mac Gregor, K. B. 2009, ApJ, 696, 1823

Gilroy, K. K. 1989, ApJ, 347, 835

Gilroy, K. K. \& Brown, J. A. 1991, ApJ, 371, 578

Gratton, R. G., Carretta, E., Matteucci, F., \& Sneden, C. 2000, A\&A, 358, 671

Guandalini, R., Busso, M., Ciprini, S., Silvestro, G., \& Persi, P. 2006, AËA, 445, 1069

Guandalini, R. \& Busso, M. 2008, A\& $A$, 488, 657

Guandalini, R., Palmerini, S., Busso, M., \& Uttenthaler, S. 2009, PASA, 26, 168

Guandalini, R. 2010, $A \mathscr{E} A$, in press

Kraft, R. P. 1994, PASP, 106, 553

Nordhaus, J., Busso, M., Wasserburg, G. J., Blackman, E. G., \& Palmerini, S. 2008, ApJ (Letters), 684, 29

Palacios, A., Charbonnel, C., Talon, S., \& Siess, L. 2006, A\&AA, 453, 261

Palmerini, S. \& Busso, M. 2008, New AR, 52, 412

Palmerini,S., Busso, M., Maiorca, E., \& Guandalini, R. 2009, PASA, 26, 161

Palmerini, S. et al. 2010, this conference

Sweigart, A. V. \& Mengel, J. G. 1979, ApJ, 229, 624

Uttenthaler, S., Lebzelter, T., Palmerini, S., Busso, M., Aringer, B., \& Lederer, M. T. 2007, $A \mathscr{E A}$ (Letters), 471,41

Wasserburg, G. J. \& Busso, M. 2008, AIPC, 1001, 295 